TITLE

AUTHOA(S)

SUBMITTED TO
REMOTE SENSING APPLICATIONS OF THE EXTENDED RADIOSITY METHOD

S.A.W. Gerstl and C.C. Borel

1992 International Geoscience and Remote Sensing Symposium 9IGARSS ' 92). Houston, TX 26-29 May 1992 and IGARSS 192 GRSS DIGEST

\section{DISX'LAIMER}

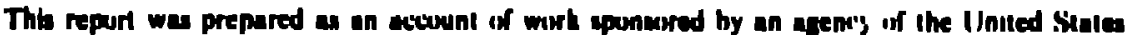

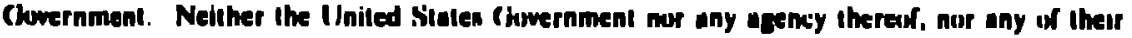
emphoyeer, inates any warraniy, express of implied, of asuumes any lepal linbalily of renponsl.

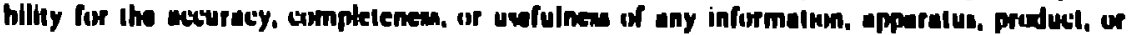

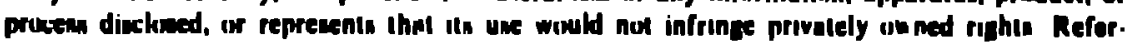

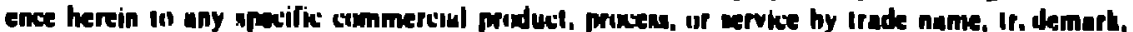

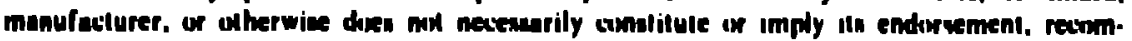
mendatum, or lavoring hy the I/nited Siates (ivvernment of nny asency therear The vrews

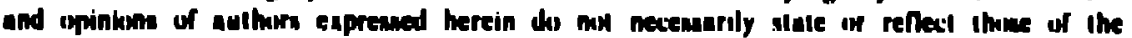
Inited Stules (komernment of any aponcy hereat.
\end{abstract}

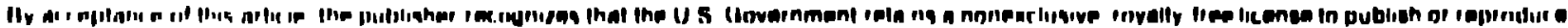

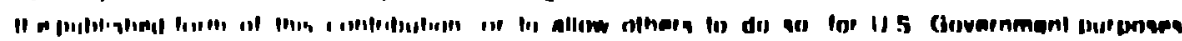

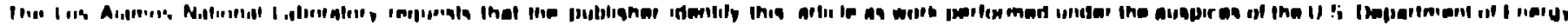




\title{
REMO'TE SENSING APPLICATIONS OF THE EXTENDED RADIOSITY METHOD
}

\author{
S.A.W. Gerstl and C.C. Borel \\ Space Science \& Technology Division \\ Los Alamos National Laboratory, MS A-104 \\ Los Alamos, New Mexico 87545, USA
}

\begin{abstract}
In this paper we describe the progress made in the last three years on developing the radionity method for remote sensing applications. The research covered canopy modeling, volumetric acat. tering and atmospheric corrections for future analysis of EOS imaging spectrometer data.
\end{abstract}

\section{Introduction}

Within NASA'a Remole Sensing Science (RSS) program the au. thors have studied applicationa of the radiosity method in re. ote senaing. The first application was in modeling the cuat. tering of light inaide vegetative canopies. We developed a program to olve the radiosity equations for the acattering of light from many acattering leaves above a ground aurface. Uaing ray. tracing, we developed inethode to compute the bidirectional re flectance distribution function (BRDF) of canopies. We alaw conducted reflectance measurementı on an artificial plant canopv composed of 12000 circular diske that airnulate leaves. The meaourements ohowed good agreement with the rartiosity calcula. tion. The second application was to model the acattering be tween a curface and a acattering or volumetric medium to rep. resent an atmonphere. A fiat ourface with a reflectance map was placed under $\bullet$ uniformly acattering medium and the ourface and volume radionitiea were compuled and then rendered for a xiven view direction. Third, from this extended radiosity method we were able to formulate a method to compute the point oprend function for any view direction and with height-dependent at. mospheric ocaltering parameters and phee func' ons. Uaing an inverse filtering method we were able to sharpen images blurred by the adjacency effect. 'The method involved the deconvolution of une meaured radiances with an inverse point apread function. Fourth, we are currently developing hyperopectral applications of the radionity method for H.OS. oensed vegetated and bare our. faces with imaging opectrometero.

\section{Canopy Modoling}

Initially we introduced the radionity mothod to moule the the multiple acaltering and tranomionion in plant ranopies. We tir. valoped a radionity code for a canopy composed of circular diace placed in $N$ layers with $M$ diece ench. Fach diac 1 in dencribed by the center location $\left(x_{1}, y_{1}, x_{1}\right)$, the normal vestor ri, and ratiua $r_{1}$. The view factore are compuland by raytracing and a large view factor matrix with $(N \times M I)^{\prime}$ elements is atored in a file. We per formed radionity calculations for various canopy geometrins wit! up to 9,000 diecs in 0.5 to 11.5 hours of CPU time on a VAX 8600 . Mure recently we solved a radiosity problem for 14400 disks in 21.73 hourn on a small workstation (SUN SPARCsta. tion IPC). The 6 million view factors where stc, ed in compressed form uning $24 \mathrm{MB}$ of diak space. The conceptual principles of the radiosity method were described in $(1)$; the algorithm is de. scribed in detail in (4). Methods to include akylight were derived and implemented. A layer-based radiusily program was devel. oped to run on an IBM PC.AT. Bidirectional reflectance distri. bution functions (BRDF) were computed for various illumina tion and observation conditions using a raytracing program. The influence of okylight and varying leaf reflectance/tranamittance values on the angular signalure was investigated

Next we worked on verifying the radionity solution with the help of a theoretical modei and measurements on an artificial plant canopy atructure. We were able to compare the radios ity solution to a clasaical radiative tranaler (RT) aolution for canopice with horizonial leaves. Analytical expreanion a well a numerical resulto were compared and found to ;ree per. fectly. By computing the up. and down-fluxes at ach layer uning weighted oume of radiunities of other lnyess, we derived that the radionity equations lead to the clanical RT differential equation for the limit of a continuous canopy atructure, if $|5|$ An intereating effect of enhanced brightness un eunlit diacs in lower layers for highly reflective discs $(p ; 0)$ was predicled uoing the rationity method and experumentally verified on our aimulated ortificial canopy.

'The radiosity method for problemo with many surfaces takes much computer time and therefore, we tried to develop aimpler, though atill radionity baed plant canopy modelo. The oimpli. fication is posable when leaf layers are comadered rather than individual leaves. We expanded a N layee model lo include Ieal layera with inslined leaves and computed view farture between layero uning - Monte Ciarlo appronch. Bnce the resulting in dionity equations are onlved we use olandard probinbility based canopy modela to compute the HIRI)F"' an illuatinted in |4|. 


\section{Applications of the Extended Ra- diosity to Atmospheric Corrections}

The extended radiosity method consists of two coupled systems of equations $\{2,3\}$ when applied to a combined canopy/atmosphere problem. The first one describes the radiosity $B_{i}^{0}$ coming from a surface $A_{i}$ as the sum of the emitted radiative flux $E_{i}^{\prime}$, the refiected fraction of radiosities from all visible surfaces and the reflected radiosities from all visible volume elements. The second equation expresses the flux density leaving a volume $V_{k}$ as the sum of the emitted flux and the scattered fractions of ra. dionities from all visible volume elements and surface elements. The fractions of reflected or scattered radiosities are the view factors. In Figure I we show a symbolic representation of the extended radionity method.

We implemented the extended radiosity method $|3|$ and have used it to simulate atmospheric scaltering over an inhomogeneous reflecting surface. We were able to rsproduce the adjacency effect from rendering the radiosity solution. One ma jor drawback of the presently implemented code is that it re. quires a large number of multiplications for each iteration in a Gauss-Seidel method. We think that adaptive meshing tech. niques, i.e. fine space subdivision near the ourface and then more coarse subdivision further away will improve the compu. tation time arastically. We believe that the extended radiositv method will provide a general purpose simulation tool for remote senuing applirations which is $a b l=$ to incorporate surface. surface, surface-volume and volume-surface effects using com puter graphica methoda. The radionity alution is global i.e. for a given illumination condition any view of the scene or inside the scattering volume can be rendered. Other mithods such as the Monte Carlo method require new oolutione for changiag view angles. Thus by taking advantinge of the global illumination calculation, effects of view angles and observer positions can be atudied. Radiosity solutions can be oblajned for any wavelength band in the visible and near infrared.

We also developed a method to compute the point apreal function uning part of the extended radionity equationa. The point apread function $P S F\left(x, y, z ; x_{0}, y_{0}, z_{0} ; \theta_{r}, \phi_{n}\right)$ is defined an the ocallering contribution of a ourface element $d A-d x d y$ l, cated at $(x, y, z)$ into the line-of-aight diraction of the observer $\left(\theta_{r}, \phi_{r}\right)$ looking al point $\left(x_{0}, y_{0}, z_{0}\right)$. One can model the blurrink effect due to the adjacency elfect with a point spread function (PSF). This PSF is a filtor finction which is convolved with the unperturbeci (no atmophere) image of a suifare. We deacribe thie wotk in more detail in these proceedings |2|. Uaing an in. verue filterit s method it is pounible to compute an inverse pont upreno function which when convolved with the menured radi ance image reducen the adjocency effect conaiderably, which in equivalent to an atmoupheric correction algorithm.

\section{Modoling Torrnin Effrets}

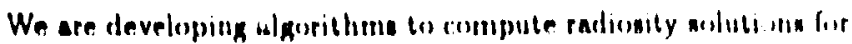
digital terrain models (ourfaic ourface acaltonting) and ate trymk (1) include BRDFH in our radionity calculationn uning apherical

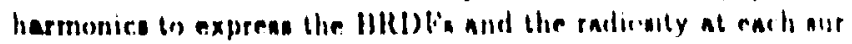
face.

\section{Future Work}

We are now in the process of integrating these various applica. lions of the radiosity method into a simulation program which computes the surface-surtace, surface-volume, volume-surface and volume-volume interactions that are of importance in remote sensing. This work will probably done on a massively parallel computer like the Connection Machine (CM-2 or CM5).

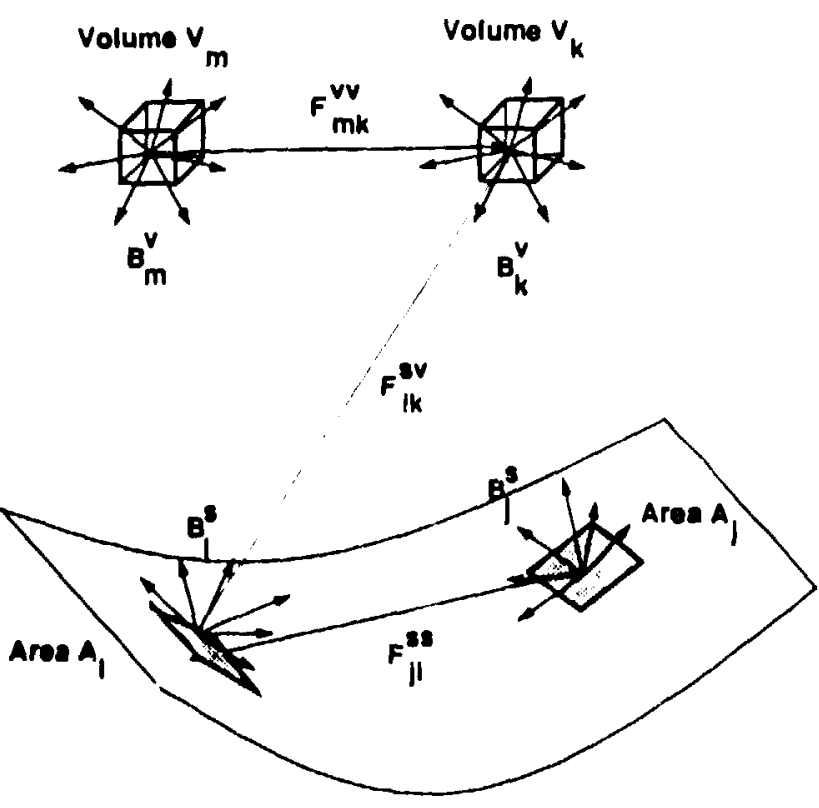

Figure 1: Visualization of the extended radiosity method for a remote sensing application including atmospherie and topogra. phy effecto.

\section{References}

(1) S.A.W. Cientl and C.C. Borel. "Principles of the radionity method for canopy reflectance modeling", Proc. Int. (ieo. science and Remote Sensing Symposium, May 20.21, 1990. Washington D.C., 3.1735 1737, 1990.

12; C.C. Borei, S. A.W. Cierotl. "A:moapheric Correctione uning the Volume Radionity Method", Iroc Int Cieoscience and Ramote Sensing Symposium, May 86 29, 1998. Kouston. Tezas, 1992

(3) C:C Horel, $\& \wedge$ W (ierotl "Simulation of partially ib" scured acenes uning the radionity method", strlt; lobl 1686. pp.271 277, April 1991

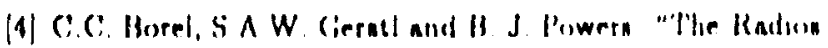
ity Mothod in Gptical Kemote Senmung of Strunbured 311 Surfaces", Remole siensing of the tinureonment, 3ti 1344. 1801

(5) J. Hoan. The Kadiation Regime and Architerture of Illant

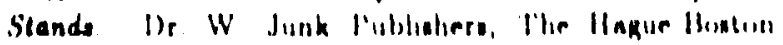
london, I9HI 